\title{
SYNTHETIC MINORITY OVERSAMPLING TECHNIQUE AND FRACTAL DIMENSION FOR IDENTIFYING MULTIPLE SCLEROSIS
}

\author{
YU-DONG ZHANG \\ School of Computer Science and Technology, Nanjing Normal University \\ Nanjing, Jiangsu 210023, P. R. China \\ Hunan Provincial Key Laboratory of Network Investigational Technology \\ Hunan Policy Academy, Changsha, Hunan 410138, P. R. China \\ yudongzhang@ieee.org \\ YIN ZHANG \\ School of Information and Safety Engineering \\ Zhongnan University of Economics and Law, Wuhan \\ Hubei 430073, P. R. China \\ yin.zhang.cn@ieee.org \\ PREETHA PHILLIPS \\ West Virginia School of Osteopathic Medicine \\ 400 N Lee St, Lewisburg, WV 24901, USA \\ pphillips@osteo.wvsom.edu \\ ZHENGCHAO DONG \\ Translational Imaging Division \& MRI Unit \\ Columbia University and New York State Psychiatric Institute \\ New York, NY 10032, USA \\ zd2109@cumc.columbia.edu
}

*Corresponding author.

This is an Open Access article published by World Scientific Publishing Company. It is distributed under the terms of the Creative Commons Attribution 4.0 (CC-BY) License. Further distribution of this work is permitted, provided the original work is properly cited. 


\author{
SHUIHUA WANG* \\ Department of Electrical Engineering, The City College of New York \\ CUNY, New York, NY 10031, USA \\ Department of Mechanical and Control Engineering \\ Kyushu Institute of Technology \\ Fukuoka Prefecture 804-8550, Japan \\ wangshuihua@njnu.edu.cn
}

Received January 13, 2017

Revised March 22, 2017

Accepted April 1, 2017

Published June 2, 2017

\begin{abstract}
Multiple sclerosis (MS) is a severe brain disease. Early detection can provide timely treatment. Fractal dimension can provide statistical index of pattern changes with scale at a given brain image. In this study, our team used susceptibility weighted imaging technique to obtain 676 MS slices and 880 healthy slices. We used synthetic minority oversampling technique to process the unbalanced dataset. Then, we used Canny edge detector to extract distinguishing edges. The Minkowski-Bouligand dimension was a fractal dimension estimation method and used to extract features from edges. Single hidden layer neural network was used as the classifier. Finally, we proposed a three-segment representation biogeography-based optimization to train the classifier. Our method achieved a sensitivity of $97.78 \pm 1.29 \%$, a specificity of $97.82 \pm 1.60 \%$ and an accuracy of $97.80 \pm 1.40 \%$. The proposed method is superior to seven state-of-the-art methods in terms of sensitivity and accuracy.
\end{abstract}

Keywords: Synthetic Minority Oversampling Technique; Canny Edge Detector; Fractal Dimension; Minkowski-Bouligand Dimension; Box Counting; Three-Segment Representation; Biogeography-Based Optimization.

\section{INTRODUCTION}

Among all various brain diseases, multiple sclerosis $(\mathrm{MS})^{1+3}$ damages the insulating covers of neural cells. The symptoms include mental, psychiatric, and physical problems. $\stackrel{4}{*}$ The symptoms may disappear between attacks. ${ }^{5}$ Currently, treatments are provided to improve patients' functioning. The life expectancy is about $5-10$ years,$\frac{6}{6}$ lower than unaffected people.

Diagnosis of magnetic resonance imaging (MRI) is usually based on scanning of MRI. However, a challenge arises due to the "normal-appearing white matter (WM)" problem, 7 which causes the lesions within the WM to appear the same as healthy WMs $\stackrel{8}{\text { Even }}$ experienced neuroradiologist may not perceive the differences.

The computer vision $(\mathrm{CV})^{9[10}$ has shown superior power to human beings in many fields. Besides, scholars have already used CV in MS detection. For example, Wang and $\mathrm{W} \mathrm{u}^{11}$ offered an adaptive chaotic particle swarm optimization (ACPSO) method. Murray, Rodriguez and Pattichis ${ }^{12}$ proposed a novel multiscale amplitudemodulation frequency-modulation (MAMFM) method. Nayak et al.$^{13}$ presented a new abnormal brain classifier on the basis of two-dimensional discrete wavelet transform (2D-DWT) and random forest (RF). Zhan and Chen ${ }^{14}$ employed biorthogonal wavelet transform (BWT) and logistic regression (LR). Yang 15 utilized $\mathrm{Hu}$ moment invariant (HMI). Zhou ${ }^{16}$ employed stationary wavelet entropy (SWE). Their classifier used decision tree (DT) and support vector machine (SVM). Siddiqui et al ${ }^{[17}$ proposed an automated and intelligent medical decision support system (AIMDSS). Phillips et al! ${ }^{18}$ combined wavelet entropy (WE). They also presented a novel hybridization method to train the classifier. 
Fractal dimension (FD) is a relatively new image feature used in $\mathrm{CV}$, and it has been successfully applied in coal $[19]$ structure rebuilding, 20 delamination detection, 21 etc. In this study, we make a tentative test to use FD to extract distinguishing features from MS brain images.

Classifier establishment is another important problem. After investigation and analysis to current classifiers, we decided to use single hidden layer neural network (SHLNN) because of the universal approximation theory. The training of classifier may suffer from massive problems: local minimum points, vanishing and exploding gradient, etc. These problems can be easily solved by swarmintelligence training algorithms, which can train weights and biases of different classifiers. For example, Arabasadi et al.,22 used genetic algorithm (GA) to train the neural network. Ji23] employed particle swarm optimization (PSO). Bahrami and Ardejani24 employed simulated annealing (SA) to train artificial neural network. Yang 25 utilized biogeography-based optimization (BBO), and their simulation results validated the superior performances of $\mathrm{BBO}$ to other algorithms.

Nevertheless, the GA method in Ref. 22 may fall in local flat areas, and the classifier may not work well. The PSO in Ref. 23 is an old algorithm. The $\mathrm{SA}$ in Ref. 24 only contains the stochastic idea, and does not include the cooperation concept. In this study, we chose the $\mathrm{BBO}$ and proposed two improvements to further enhance its performance.
The structure of the remainder is organized below: Section 2 gives the source of materials. Section 3 describes the rationale of the methodology. Section 4 presents the results, and discusses the results. Section 5 concludes the paper.

\section{MATERIALS}

We acquired 676 slices from $38 \mathrm{MS}$ patients from the website of University of Cyprus. All those slices contain plaques. Besides, we acquired 880 brain slices from 34 healthy controls in China local hospitals. The details of both MS patient and healthy subject are described in Ref. 16.

\subsection{Histogram Stretching}

Since the dataset sources are from different countries, we employed the histogram stretching (HS 2 method to ease slice comparability. There are other advanced image enhancement techniques, such as local adaptive histogram equalization method, 27 pulse-coupled neural network method, $\stackrel{28}{28}$ local graylevel transformation, 29 deep autoencoder method,, 30 etc. Nevertheless, the HS is efficient for its simple and easy to implement. Practically, we increased the dynamic range of all images to the same level. Assume $c$ denotes original brain slice, $d$ the HS normalized slice, we have

$$
d(x, y)=\frac{c(x, y)-c_{\min }}{c_{\max }-c_{\min }},
$$

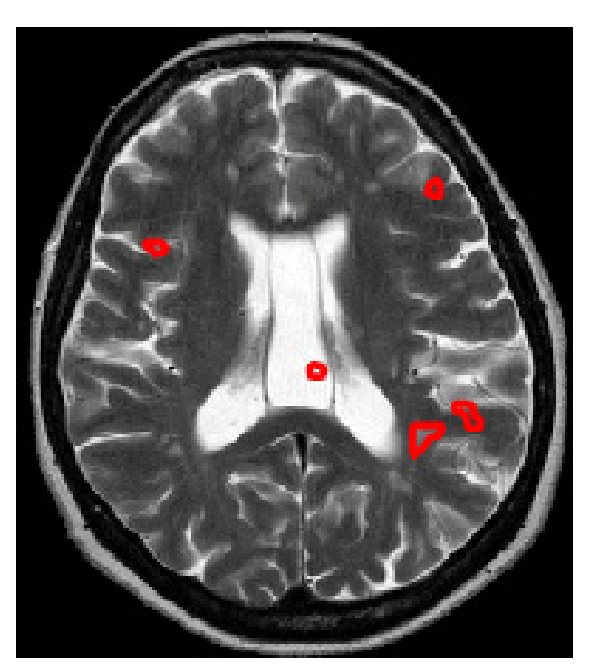

(a)

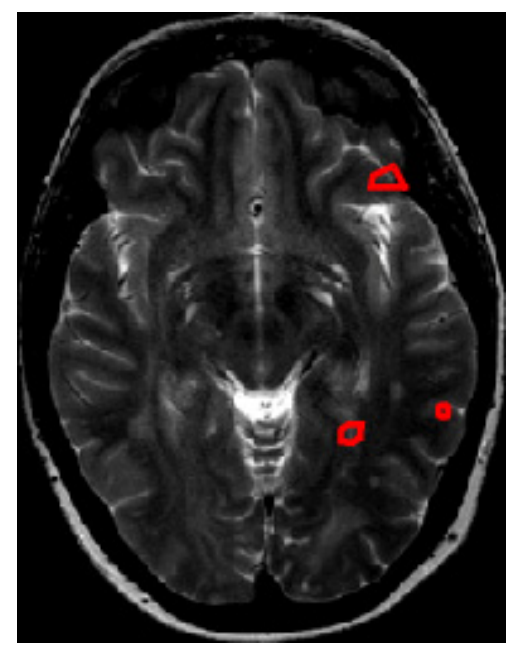

(b)

Fig. 1 Sample of our dataset. 


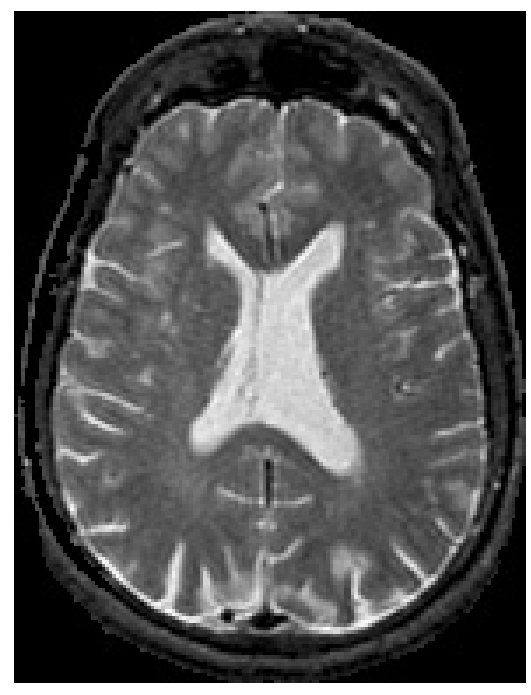

(c)

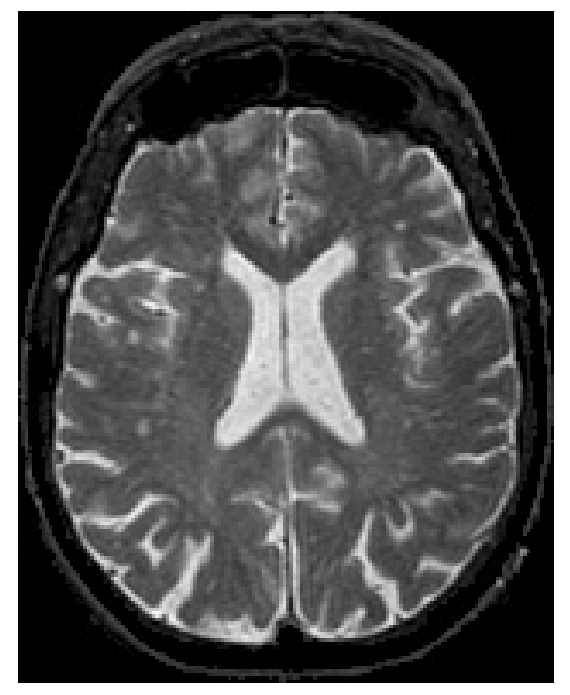

(d)

Fig. 1 (Continued)

where $x$ denotes the horizontal position and $y$ the vertical position. The $c_{\min }$ and $c_{\max }$ are defined as

$$
\begin{aligned}
c_{\min } & =\min _{(x, y)} c(x, y), \\
c_{\max } & =\max _{(x, y)} c(x, y) .
\end{aligned}
$$

The first row in Fig. 1 shows two MS slices with five plaques and three plaques, respectively. The last row in Fig. 1 shows two healthy slices. All the slices are extracted along axial direction with different slice index.

\subsection{Synthetic Minority Oversampling Technique}

As the number of healthy slices (880) is slightly larger than the number of MS slices (676), we used Synthetic Minority Oversampling Technique (SMOTE) 31 to increase the number of MS slices. Undersampling method is not used, since it will reduce the size of the dataset. We first take a sample $I$ from the minority class, and select its $k$ nearest neighbors. We select one neighbor $J$ from the $k$ neighbors. We then draw a vector $V$ from $I$ to $J$.

$$
V=J-I \text {. }
$$

Finally, a new synthetic sample $S$ is generated as

$$
S=I+\alpha \times V,
$$

where $\alpha$ is a random number between 0 and 1, obeying the uniform distribution.

Using SMOTE method, we generated 204 synthetic MS samples. In total, we have 880 healthy slices and $880 \mathrm{MS}$ slices. Thus, our dataset contains 1760 image.

\section{METHODOLOGY}

\subsection{Canny Edge Detection on Brain Slices}

The FD is conventionally based on the binary image; hence, scholars usually combine edge detection with FD. The edge detection aims to identify the edges where brightness has discontinuity. There are many edge detection methods. Canny edge detector (CED) ${ }^{32}$ is the most effective one, and widely used in FPGA architecture,,$\underline{33}$ remote sensing, etc. The procedures of CED are listed below in Table 1 .

Figure 2 shows the comparison of different edge detectors over a hand image. Figure $2 \mathrm{a}$ shows the original image. Figures $2 \mathrm{~b}-2 \mathrm{f}$ provide the edge detection results by Laplacian of Gaussian (LoG), Robert edge detector (RED), Prewitt edge detector (PED), Sobel edge detector (SED) and CED, respectively. Obviously, the CED results contain the most abundant texture information among all edge detectors. Besides, CED did not remove any distinguishing features.

\subsection{FD Model}

FD is widely used for image encoding, 3435 image segmentation, $\frac{36}{,}$ image classification, $\stackrel{37}{,}$ cloud computing, ${ }^{38}$ etc. Assume $F$ denotes the FD, $S$ the scaling factor, $M$ the number of sticks for measuring, 
Table 1 Procedures of CED.

Step 1 Use Gaussian filter to remove noises and smoothing

Step 2 Calculate and locate the intensity gradients

Step 3 Use non-maximum suppression to eliminate spurious edges

Step 4 Employ double threshold to find potential edges

Step 5 Edge tracking based on hysteresis

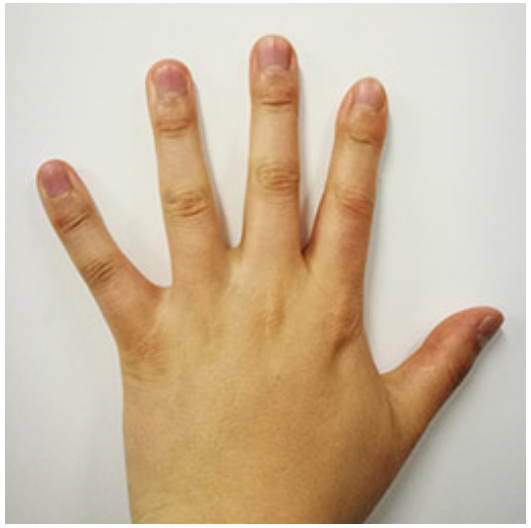

(a) Original Image

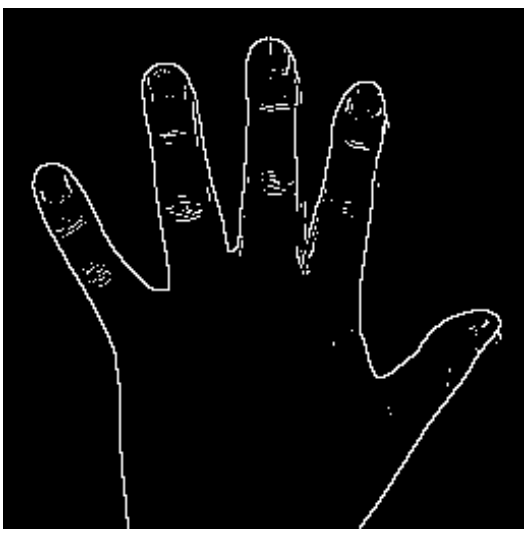

(c) RED

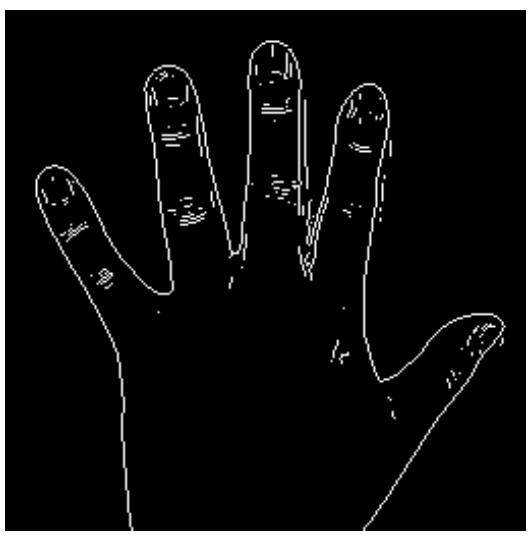

(e) SED

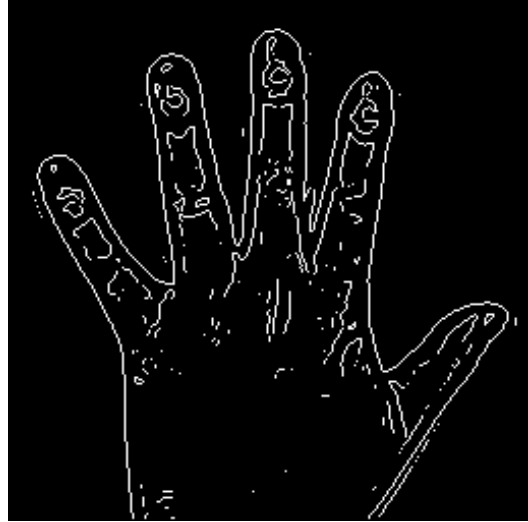

(b) LOG

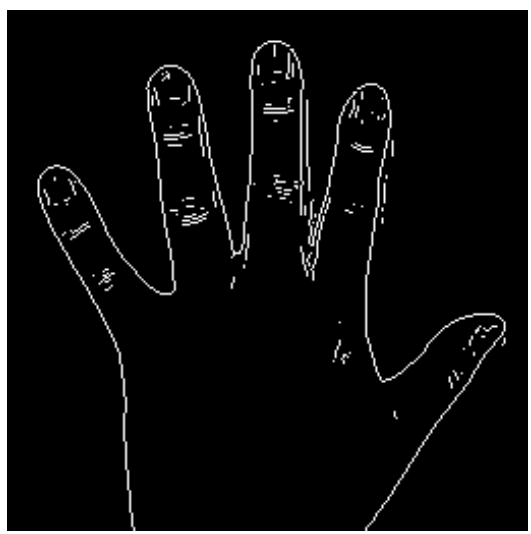

(d) PED

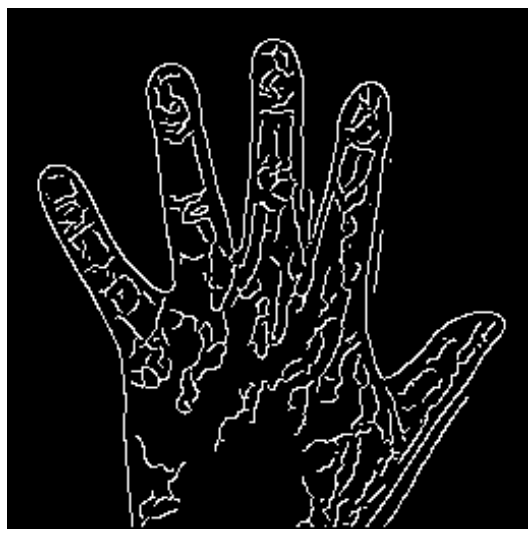

(f) CED

Fig. 2 Comparison of different edge detectors (LOG = Laplacian of Gaussian, RED = Robert edge detector, PED = Prewitt edge detector, $\mathrm{SED}=$ Sobel edge detector, $\mathrm{CED}=$ Canny edge detector ) 


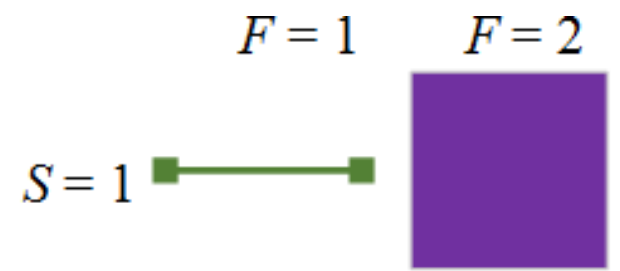

(a)

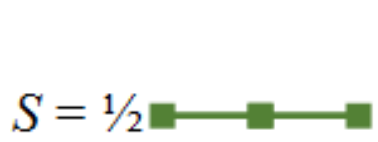

(d) (b)

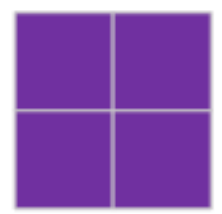

(e)

$$
F=3
$$

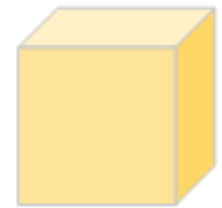

(c)

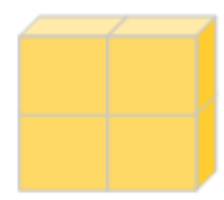

(f)

Fig. 3 Relationship among dimension and geometry.

we can deduce the relationship among those three variables:

$$
M=\left(\frac{1}{S}\right)^{F} .
$$

This scaling rule is coherent with traditional concepts between dimension and geometry. ${ }^{3940}$ Afterwards, we extend above formula to general field as

$$
F=-\log _{S} M \text {. }
$$

Figure 3 shows several samples. The number of sticks $M$ used to cover the shape is $M=1$ for Figs. 3 a 3r. For the second row, $M=2,4$ and 8 for Figs. 3 $\mathrm{d}-3$; , respectively. We can observe the relationship is in line with Eqs. (6) and (77).

Another example is the Koch curve ${ }^{41}$ with FD $F$ of 1.2619. Figure 4 shows the Koch curve. Figure 4 a shows the curve with iteration $I=1$. Figure 40 shows the curve with $I=2$ and so forth.

\subsection{Minkowski-Bouligand Dimension}

Section 3.2 shows the theoretical model of FD. In practice, scholars usually used MinkowskiBouligand dimension (MBD) $)^{42}$ to estimate the FD for a given image. MBD was laid over a regular grid over the image and the number of boxes counted overlapped with the edges.

The grid scale $G$ in this study was assigned with the value of $16,8,4,2$ and 1 in sequence. Therefore, we shall get a five-element vector as the feature space. Figure 5 shows the hand image covered by boxes with size $G$ of 8 . The fractional calculus methods 4345 shall be considered in the future.

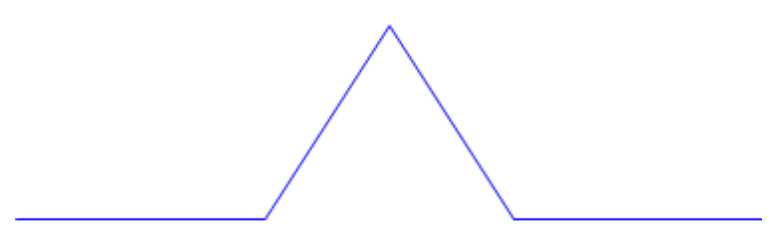

(a) $I=1$

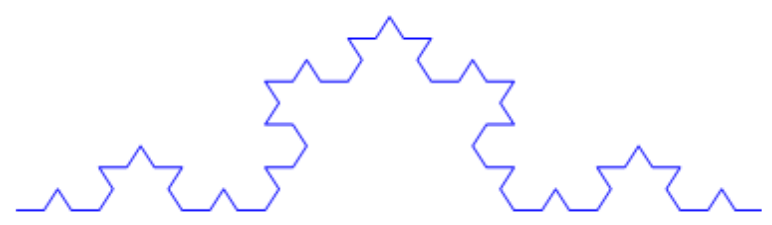

(c) $I=3$

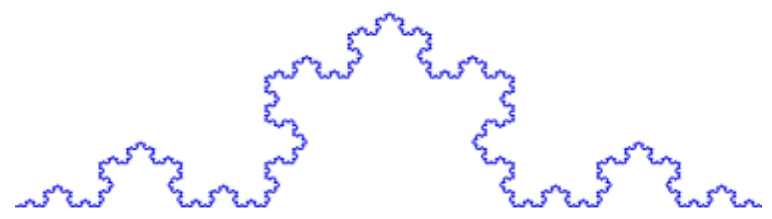

(e) $I=5$

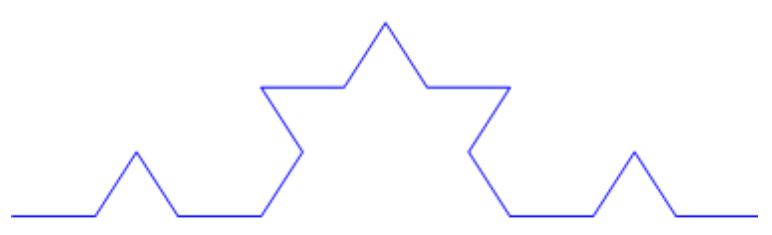

(b) $I=2$

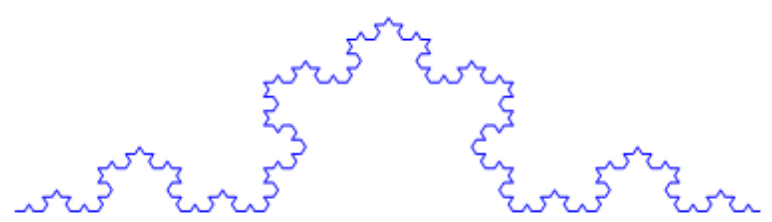

(d) $I=4$

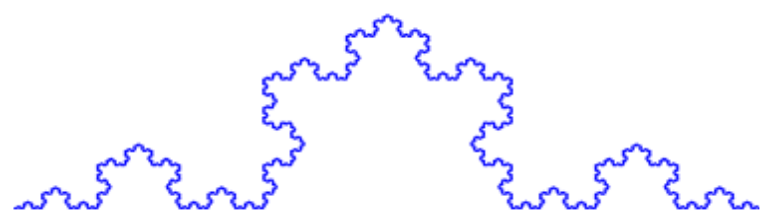

(f) $I=6$

Fig. 4 One to six iterations of Koch curve $(F=1.2619)$. 


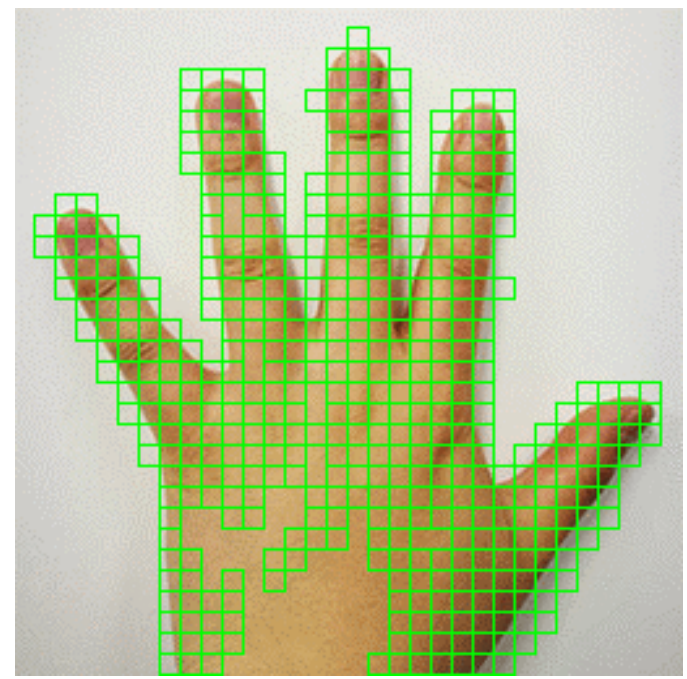

Fig. 5 The hand image covered by boxes with size $G$ of 8 .

\subsection{Single Hidden Layer Neural Network}

Theoretically, the SHLNN can approximate to any function due to the universal approximation theory: Any SHLNN with a finite number of neurons can approximate continuous functions on compact subsets of Euclidean space, under mild assumptions of the activation functions. This advantage makes us not only choose SHLNN but also other classifiers, including extreme learning machine, 46147 linear regression classifier, $\underline{48}, \underline{50}$ support vector machine, $\stackrel{51-53}{5 T}$ DT,$\underset{54}{ }$ Bayesian classifier,$\frac{55}{}$ etc. There are too many literatures discussing the concept and structure of SHLNN ${ }^{56 / 57}$ In this paper, we shall briefly introduce its fundamentals.

Suppose there are $d$ different classes and $f$-dimensional features. Suppose there are $Z$ labelled samples, we have the $z$ th training data as

$$
x(z)=\left[x_{1}(z), x_{2}(z), \ldots, x_{f}(z)\right]^{T} .
$$

It has a corresponding label

$$
L(z) \in[1,2, \ldots, d] .
$$

The output of the trained classifier with input of $\mathbf{x}(z)$ is

$$
y(z)=\left[y_{1}(z), y_{2}(z), \ldots, y_{d}(z)\right]^{T} .
$$

The predicted label of $\mathbf{x}(z)$ is

$$
P(z)=\arg \max (y(z)) .
$$

The criterion of the SHLNN is to minimize the mean-squared error between predicted label $P(z)$ and realistic label $L(z)$ as

$$
\min \sum_{z=1}^{Z}(P(z)-L(z))^{2} .
$$

\subsection{Transform from Training to Optimization}

To establish the SLHNN classifier, we need to train the weights and biases. Besides, there is an important parameter - the number of hidden neurons, should be optimized. In this study, we transform the above two problems to an optimization, which can accomplish the above two tasks simultaneously.

The idea is the three-segment representation (TSR) inspired from Ref. 42, For any swarmintelligence algorithm, we divide the solution candidate into three segments. Segment 1 (S1) is encoded as the weights, Segment 2 (S2) is encoded as the biases, and the rest Segment 3 (S3) is encoded as the number of hidden neurons, as shown in Fig. 6 .

\subsection{TSR-BBO}

The TSR was then embedded into the BBO. The combined method is named TSR-BBO. The BBO is a hot swarm intelligence algorithm that has been widely used in cost optimization,, 58 fruit classification,, 59 sonar classification, 60 etc.

In the BBO, each habitat (i.e. solution candidate) is measured by a fitness function named "habitat suitability index (HSI)". The HSI of habitats relies on the variables named as "suitability index variables (SIV)" ${ }^{61}$ In this study, the SIV is encoded by the three-segmentation representation way.

Three important procedures are included in BBO algorithm. They are (i) migration, (ii) mutation and (iii) elitism. In what follows, we shall introduce them briefly.

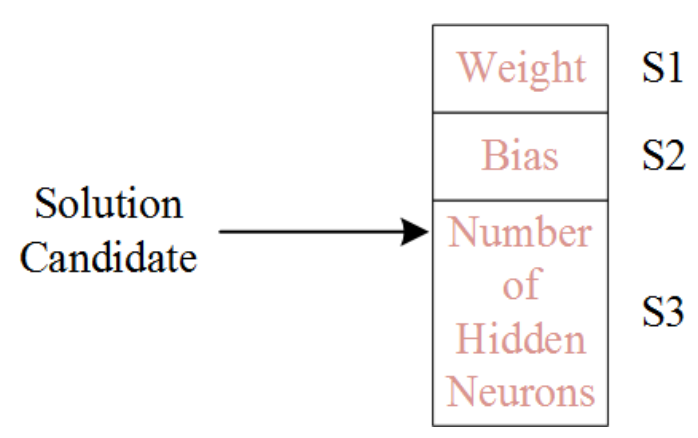

Fig. 6 TSR. 


\section{Y.-D. Zhang et al.}

Solution candidates with larger HSI are more possible to emigrate; in contrast, candidates with smaller HSI are more possible to immigrate. Let $s$ denote the number of species, and $S$ the maximum number of $s$. Assume the emigration rate is $w$ and immigration rate is $v$, their relationship is depicted by the following equations:

$$
\begin{aligned}
& w(s)=W \times \frac{s}{S}, \\
& v(s)=V \times \frac{1-s}{S},
\end{aligned}
$$

where $W$ denotes the maximum value of $w$ and $V$ the maximum value of $v$.

Mutation aims to increase the diversity of the ecosystem ${ }^{62}$ Suppose $n$ is the mutation rate, $N$ is the maximum mutation rate. We further assume $r(\mathrm{~s})$ denotes the solution probability of species $s$, $R$ is the maximum value of $r$. We can deduce that

$$
n(s)=\frac{1-r(s)}{R} \times N .
$$

In the view of SIV, the mutation was implemented on a rand number generator $c$, which follows uniform distribution in the range of $[0,1]$. Suppose a candidate at $k$ th step $\mathbf{Q}_{k}$ is

$$
Q_{k}=\left[Q_{1, k}, Q_{2, k}, \ldots, Q_{i, k}, \ldots, Q_{I, k}\right] .
$$

Here, $Q_{i, k}$ represents the $i$ th SIV value at $k$ th step, and $I$ the dimension of solution space. The temporary mutated variable $M$ is performed as

$$
M=Q_{i, k}+c \times(U(i)-L(i)),
$$

where $U(i)$ and $L(i)$ denote the upper bound and lower bound of $i$ th SIV. $M$ is mapped to the range of $[L(i), U(i)]$ as

$$
M= \begin{cases}U(i) & \text { if } M>U(i), \\ L(i) & \text { if } M<L(i), \\ M & \text { otherwise }\end{cases}
$$

$M$ is directly assigned to $Q_{i, k}$ if the replaced candidate provides better HSI value than the original candidate.

$$
\begin{gathered}
Q_{i, k} \leftarrow M, \quad \text { if } a\left(\left[Q_{1, k}, Q_{2, k}, \ldots, Q_{i-1, k}, M,\right.\right. \\
\left.\left.Q_{i+1, k}, \ldots\right]\right)<a\left(Q_{k}\right),
\end{gathered}
$$

where $a$ represents the HSI function.

Elitism keeps the best solutions, in order to avoid them getting destructed by the mutation procedure. ${ }^{63}$ Elitism is performed by taking $v=0$ for the specified number of elites. If there is no elitism in the BBO, the optimizer will be difficult to converge.

The TSR-BBO implements the same as BBO when used to train the SHLNN, except that the former can optimize the number of hidden neurons while the latter cannot.

\subsection{The Whole System}

Our proposed MS identification method is based on successful components, including histogram stretch, SMOTE, CED, MBD, SHLNN, TSR, BBO. Its diagram is shown in Fig. 7 , The performance was measured by 10 times of 10-fold cross-validation.

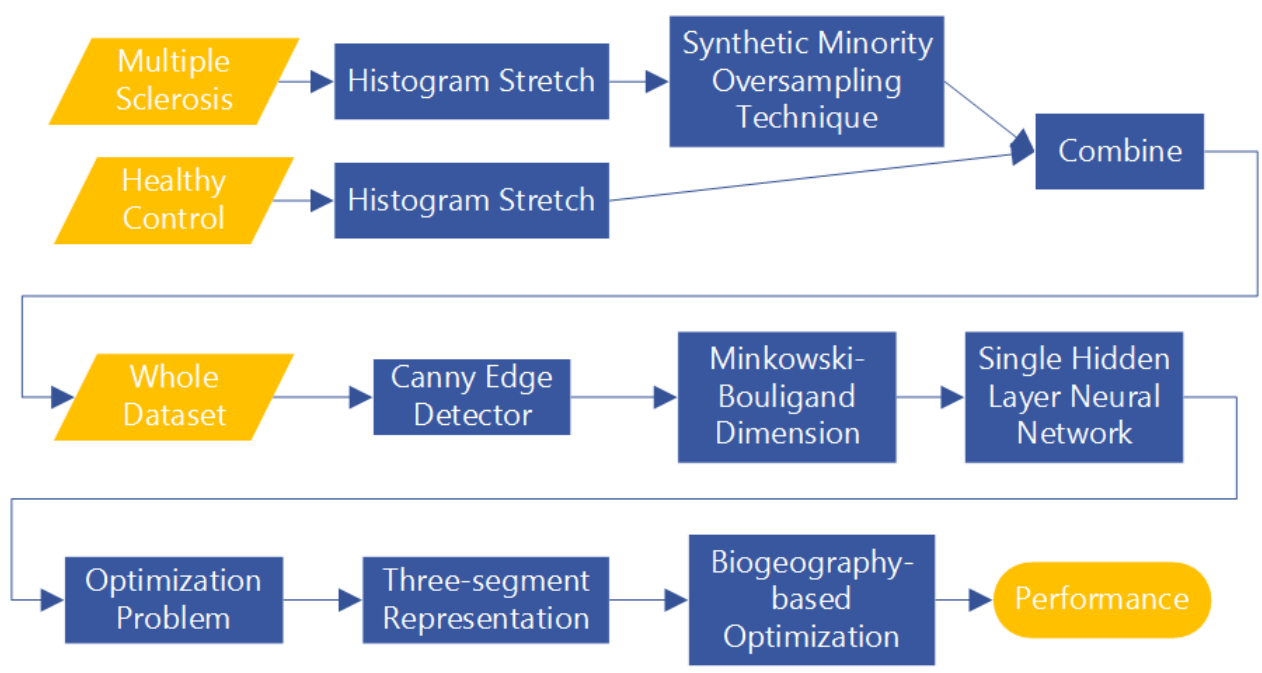

Fig. 7 Flowchart of our proposed MS identification system. 


\section{EXPERIMENTS, RESULTS AND DISCUSSIONS}

\subsection{MBD of Brain Slices}

The MBD of a MS slice is offered in Fig. 8] Figure $8 \mathrm{k}$ gives the original MS slice and Figs. $8 \mathrm{~b}-8 \mathrm{f}$ offer the MBD estimation with grid scale $G$ of $16,8,4,2$ and
1, respectively. The numbers of boxes in these five situations is presented in Table 2 .

The MBD in Fig. 8 was performed on the edges extracted by CED. Why CED performs better than other edge detectors? The reason is it contains many successful components: Gaussian filter, intensity gradient detector, non-maximum suppression,

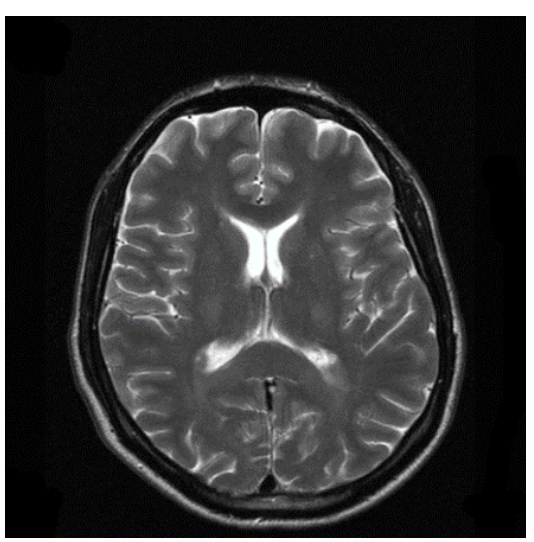

(a) Original MS slice

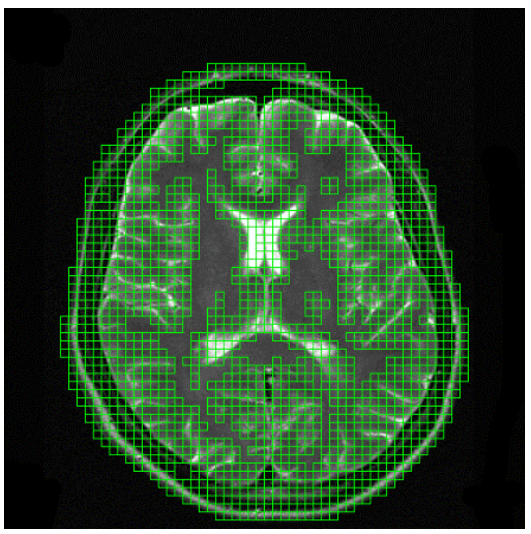

(c) $G=8$

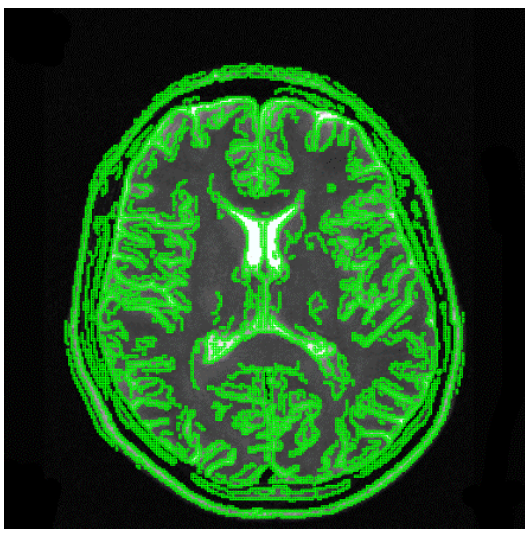

(e) $G=2$

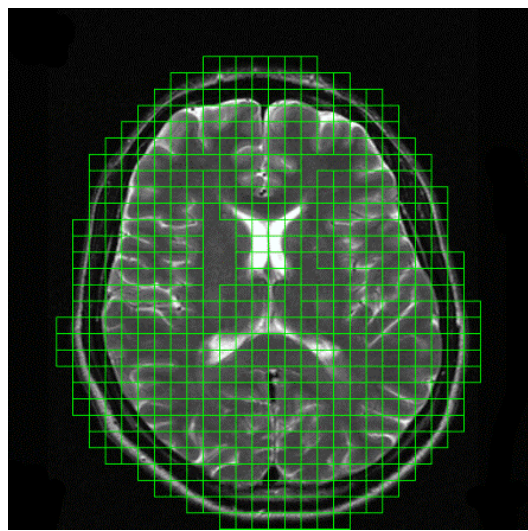

(b) $G=16$

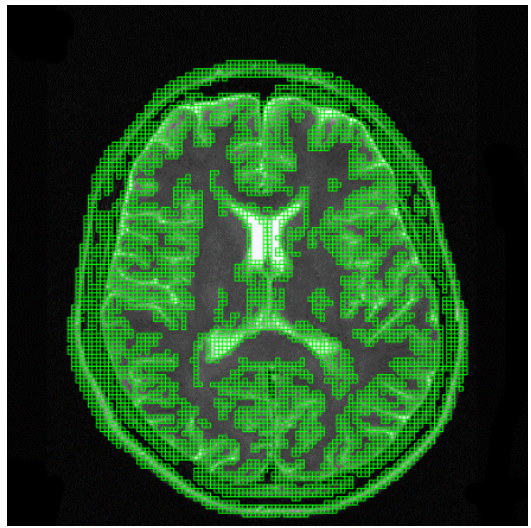

(d) $G=4$

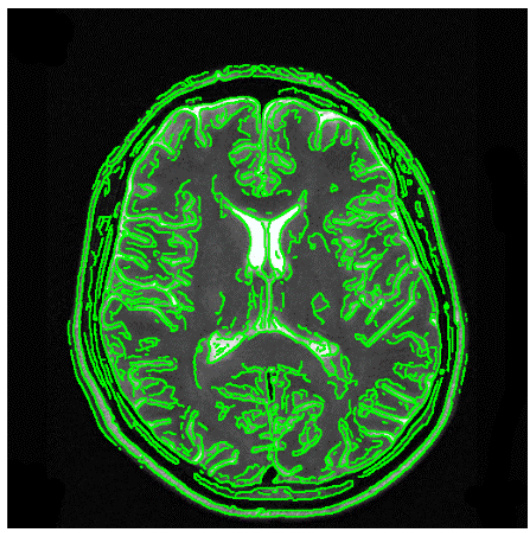

(f) $G=1$

Fig. $8 \mathrm{MBD}$ of a MS slice. 
Table 2 Number of Boxes.

\begin{tabular}{cc}
\hline Grid Scale & Number of Boxes \\
\hline 1 & 19105 \\
2 & 10333 \\
4 & 4977 \\
8 & 1776 \\
16 & 551 \\
\hline
\end{tabular}

double threshold and edge tracking. All these components help to form the effectiveness of CED.

An adaptive threshold CED was proposed by Huo and Wei.64

In the future, we shall test this adaptivethreshold CED method. A shortcoming of MBD is its computational complexity. The reason is the calculation of Euclidean distance, involving floating point number calculation. Rossales and Luppe $e^{65}$ proposed a dedicated hardware implementation scheme. We shall test this method in our future research. In the future, other advanced dimension estimation methods shall be tested. The generalized
$M$-set $[66[67$ may give equivalent or better performance than MBD.

\subsection{Statistical Analysis}

In the statistical analysis, each fold contains 88 MS slices and 88 healthy slices. The sensitivities, specificities, accuracies of our method over 10 runs of 10-fold cross validation are presented in Tables 35 , respectively. We can observe that our method achieved a sensitivity of $97.781 .29 \%$, a specificity of $97.821 .60 \%$, and an accuracy of $97.801 .40 \%$.

\subsection{Training Algorithm Comparison}

We used the proposed TSR-BBO algorithm to train the classifier. Meanwhile, the comparison basis includes GA, 22 PSO 23 SA, 24 BBO, 25 etc. The maximum iteration number is 1000 . The size of population is set to 20 . The parameters of these algorithms were obtained by experiences and are listed in Table 6 .

Table 3 Sensitivity of Our Method (Unit: \%).

\begin{tabular}{|c|c|c|c|c|c|c|c|c|c|c|c|}
\hline & F1 & F2 & F3 & F 4 & F5 & F6 & F7 & F8 & F9 & F10 & Total \\
\hline $\mathrm{R} 1$ & 95.45 & 97.73 & 98.86 & 95.45 & 98.86 & 97.73 & 96.59 & 98.86 & 97.73 & 97.73 & 97.50 \\
\hline $\mathrm{R} 2$ & 95.45 & 97.73 & 94.32 & 96.59 & 97.73 & 97.73 & 96.59 & 96.59 & 96.59 & 96.59 & 96.59 \\
\hline R3 & 95.45 & 97.73 & 95.45 & 95.45 & 97.73 & 94.32 & 95.45 & 95.45 & 96.59 & 96.59 & 96.02 \\
\hline $\mathrm{R} 4$ & 98.86 & 100.00 & 95.45 & 100.00 & 100.00 & 100.00 & 100.00 & 100.00 & 100.00 & 98.86 & 99.32 \\
\hline R5 & 97.73 & 95.45 & 97.73 & 96.59 & 96.59 & 96.59 & 97.73 & 96.59 & 96.59 & 96.59 & 96.82 \\
\hline R6 & 98.86 & 100.00 & 100.00 & 100.00 & 100.00 & 96.59 & 97.73 & 98.86 & 98.86 & 100.00 & 99.09 \\
\hline $\mathrm{R} 7$ & 96.59 & 94.32 & 94.32 & 97.73 & 95.45 & 96.59 & 97.73 & 96.59 & 96.59 & 96.59 & 96.25 \\
\hline $\mathrm{R} 8$ & 98.86 & 100.00 & 100.00 & 97.73 & 100.00 & 97.73 & 98.86 & 96.59 & 100.00 & 100.00 & 98.98 \\
\hline $\mathrm{R} 9$ & 97.73 & 98.86 & 98.86 & 98.86 & 97.73 & 100.00 & 98.86 & 98.86 & 100.00 & 98.86 & 98.86 \\
\hline $\mathrm{R} 10$ & 96.59 & 94.32 & 98.86 & 97.73 & 100.00 & 98.86 & 98.86 & 100.00 & 100.00 & 98.86 & 98.41 \\
\hline Avr & & & & & & & & & & & $97.78 \pm 1.29$ \\
\hline
\end{tabular}

Table 4 Specificity of Our Method (Unit: \%).

\begin{tabular}{|c|c|c|c|c|c|c|c|c|c|c|c|}
\hline & F1 & F2 & F3 & F4 & F5 & F6 & F7 & F8 & F9 & F10 & Total \\
\hline $\mathrm{R} 1$ & 97.73 & 98.86 & 100.00 & 97.73 & 96.59 & 94.32 & 95.45 & 97.73 & 100.00 & 98.86 & 97.73 \\
\hline $\mathrm{R} 2$ & 98.86 & 98.86 & 97.73 & 96.59 & 98.86 & 95.45 & 97.73 & 97.73 & 96.59 & 97.73 & 97.61 \\
\hline R3 & 96.59 & 96.59 & 94.32 & 97.73 & 97.73 & 96.59 & 96.59 & 98.86 & 97.73 & 96.59 & 96.93 \\
\hline $\mathrm{R} 4$ & 98.86 & 100.00 & 98.86 & 98.86 & 100.00 & 100.00 & 98.86 & 100.00 & 100.00 & 97.73 & 99.32 \\
\hline $\mathrm{R} 5$ & 95.45 & 95.45 & 96.59 & 96.59 & 96.59 & 96.59 & 95.45 & 96.59 & 96.59 & 95.45 & 96.14 \\
\hline $\mathrm{R} 6$ & 98.86 & 100.00 & 100.00 & 97.73 & 98.86 & 98.86 & 97.73 & 100.00 & 100.00 & 100.00 & 99.20 \\
\hline $\mathrm{R} 7$ & 95.45 & 94.32 & 95.45 & 95.45 & 95.45 & 94.32 & 94.32 & 95.45 & 92.05 & 92.05 & 94.43 \\
\hline $\mathrm{R} 8$ & 98.86 & 98.86 & 98.86 & 100.00 & 100.00 & 100.00 & 97.73 & 98.86 & 98.86 & 98.86 & 99.09 \\
\hline $\mathrm{R} 9$ & 100.00 & 100.00 & 98.86 & 97.73 & 97.73 & 100.00 & 97.73 & 98.86 & 98.86 & 100.00 & 98.98 \\
\hline $\mathrm{R} 10$ & 98.86 & 97.73 & 98.86 & 98.86 & 98.86 & 100.00 & 96.59 & 98.86 & 98.86 & 100.00 & 98.75 \\
\hline Avr & & & & & & & & & & & $97.82 \pm 1.60$ \\
\hline
\end{tabular}


Table 5 Accuracy of Our Method (Unit: \%).

\begin{tabular}{|c|c|c|c|c|c|c|c|c|c|c|c|}
\hline & F1 & F2 & F3 & F4 & F5 & F6 & F7 & F8 & F9 & F10 & Total \\
\hline $\mathrm{R} 1$ & 96.59 & 98.30 & 99.43 & 96.59 & 97.73 & 96.02 & 96.02 & 98.30 & 98.86 & 98.30 & 97.61 \\
\hline $\mathrm{R} 2$ & 97.16 & 98.30 & 96.02 & 96.59 & 98.30 & 96.59 & 97.16 & 97.16 & 96.59 & 97.16 & 97.10 \\
\hline R3 & 96.02 & 97.16 & 94.89 & 96.59 & 97.73 & 95.45 & 96.02 & 97.16 & 97.16 & 96.59 & 96.48 \\
\hline $\mathrm{R} 4$ & 98.86 & 100.00 & 97.16 & 99.43 & 100.00 & 100.00 & 99.43 & 100.00 & 100.00 & 98.30 & 99.32 \\
\hline $\mathrm{R} 5$ & 96.59 & 95.45 & 97.16 & 96.59 & 96.59 & 96.59 & 96.59 & 96.59 & 96.59 & 96.02 & 96.48 \\
\hline $\mathrm{R} 6$ & 98.86 & 100.00 & 100.00 & 98.86 & 99.43 & 97.73 & 97.73 & 99.43 & 99.43 & 100.00 & 99.15 \\
\hline $\mathrm{R} 7$ & 96.02 & 94.32 & 94.89 & 96.59 & 95.45 & 95.45 & 96.02 & 96.02 & 94.32 & 94.32 & 95.34 \\
\hline $\mathrm{R} 8$ & 98.86 & 99.43 & 99.43 & 98.86 & 100.00 & 98.86 & 98.30 & 97.73 & 99.43 & 99.43 & 99.03 \\
\hline $\mathrm{R} 9$ & 98.86 & 99.43 & 98.86 & 98.30 & 97.73 & 100.00 & 98.30 & 98.86 & 99.43 & 99.43 & 98.92 \\
\hline R10 & 97.73 & 96.02 & 98.86 & 98.30 & 99.43 & 99.43 & 97.73 & 99.43 & 99.43 & 99.43 & 98.58 \\
\hline Avr & & & & & & & & & & & $97.80 \pm 1.40$ \\
\hline
\end{tabular}

Table 6 Parameter Setting.

\begin{tabular}{ll}
\hline Algorithm & \multicolumn{1}{c}{ Setting } \\
\hline $\mathrm{GA}^{\sqrt{22}}$ & Crossover Probability $=0.8$, Mutation Probability $=0.05$ \\
$\mathrm{PSC}^{23}$ & Both Acceleration Factors $=1$, Inertia $=0.5$, Maximum Velocity $=1$, \\
$\mathrm{SA}^{24}$ & Initial Temperature $=100$, Final Temperature $=0$, Cooling Function $=$ Linear \\
$\mathrm{BBC}^{25}$ & Elite Number $=2$, Maximum Mutation Rate $=0.1$, Maximum Emigration $/$ Immigration Rate $=1$ \\
$\mathrm{TSR}-\mathrm{BBO}$ (Proposed) & Segment Number $=3$, Elite Number $=2$, Maximum Mutation Rate $=0.1$, \\
& Maximum Emigration/Immigration Rate $=1$ \\
\hline
\end{tabular}

Table 7 Training Algorithm Comparison in Terms of Accuracy (Unit: \%).

\begin{tabular}{|c|c|c|c|c|c|}
\hline Algorithm & $\mathrm{GA}^{22}$ & $\mathrm{PSO}^{23}$ & $\mathrm{SA}^{\sqrt{24}}$ & $\mathrm{BBC}^{25}$ & TSR-BBO (Proposed) \\
\hline $\mathrm{R} 1$ & 88.24 & 97.44 & 84.32 & 98.24 & 97.61 \\
\hline $\mathrm{R} 2$ & 91.93 & 95.57 & 85.11 & 93.92 & 97.10 \\
\hline $\mathrm{R} 3$ & 90.40 & 95.23 & 85.11 & 94.94 & 96.48 \\
\hline $\mathrm{R} 4$ & 86.31 & 97.22 & 83.13 & 96.53 & 99.32 \\
\hline R5 & 85.45 & 97.56 & 77.44 & 95.28 & 96.48 \\
\hline $\mathrm{R} 6$ & 90.97 & 97.61 & 80.85 & 99.03 & 99.15 \\
\hline $\mathrm{R} 7$ & 92.27 & 98.24 & 85.40 & 100.00 & 95.34 \\
\hline $\mathrm{R} 8$ & 88.75 & 96.08 & 81.76 & 98.35 & 99.03 \\
\hline $\mathrm{R} 9$ & 89.60 & 97.61 & 80.57 & 98.18 & 98.92 \\
\hline $\mathrm{R} 10$ & 90.00 & 96.08 & 89.55 & 98.30 & 98.58 \\
\hline Avr & $89.39 \pm 2.25$ & $96.86 \pm 1.03$ & $83.32 \pm 3.35$ & $97.28 \pm 1.99$ & $97.80 \pm 1.40$ \\
\hline
\end{tabular}

The comparison with terms of accuracy is listed in Table 7. The boxplot is presented in Fig. 9. We can observe that the proposed TSRBBO yields the largest accuracy of $97.80 \pm 1.40 \%$, the second is the $\mathrm{BBO}^{25}$ that yields an accuracy of $97.28 \pm 1.99 \%$, the third is the PSO [23] that yields an accuracy of $96.86 \pm 1.03 \%$. The $\mathrm{GA}^{22}$ ranks the fourth with an accuracy of $89.39 \pm 2.25 \%$. The $\mathrm{SA}^{24}$ yields the worst accuracy of $83.32 \pm 3.35 \%$.

\subsection{Comparison to State-of-the-Art Methods}

Finally, our method "CED + MBD + SHLNN + TSR-BBO" was compared to seven state-of-the-art methods: MAMFM + SVM, 12 $2 \mathrm{D}-\mathrm{DWT}+\mathrm{RF}, 13$ $\mathrm{BWT}+\mathrm{LR}, \frac{14}{\mathrm{SWE}}+\mathrm{DT}, \frac{16}{\mathrm{SWE}}+\mathrm{SVM}, \frac{16}{16}$ AIMDSS 17 and WE!18

The results in Table 8 showed that our method achieved the highest sensitivity and accuracy 
Table 8 Algorithm Comparison (Unit: \%).

\begin{tabular}{|c|c|c|c|}
\hline Method & Sensitivity & Specificity & Accuracy \\
\hline MAMFM12 & 94.08 & 93.64 & 93.83 \\
\hline $2 \mathrm{D}-\mathrm{DWT}+\mathrm{RF}^{13}$ & 96.01 & 96.70 & 96.40 \\
\hline $\mathrm{BWT}+\mathrm{LR}^{14}$ & $97.12 \pm 0.14$ & $98.25 \pm 0.16$ & $97.76 \pm 0.10$ \\
\hline $\mathrm{SWE}+\mathrm{DT}^{16}$ & 96.75 & 98.30 & 97.62 \\
\hline $\mathrm{SWE}+\mathrm{SVM}{ }^{16}$ & 97.34 & 97.73 & 97.56 \\
\hline AIMDSS 17 & 95.86 & 96.48 & 96.21 \\
\hline $\mathrm{WE}^{18}$ & 96.15 & 97.16 & 96.72 \\
\hline MBD + SHLNN + TSR-BBO (Our) & $97.78 \pm 1.29$ & $97.82 \pm 1.60$ & $97.80 \pm 1.40$ \\
\hline
\end{tabular}

Note: Bold means the best.

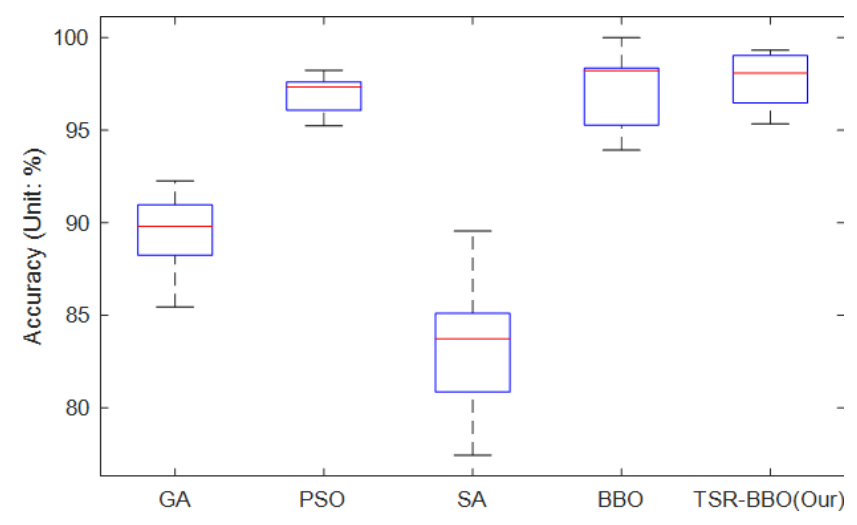

Fig. 9 Training algorithm comparison.

among eight algorithms. The highest specificity was achieved by SWE + DT ${ }^{16}$ with value of $98.30 \%$, the second highest specificity was achieved by BWT + $\mathrm{LR}^{14}$ with value of $98.25 \pm 0.16 \%$, and our method yielded the third highest specificity with value of $97.82 \pm 1.60 \%$. Concerning all three measures, our method performed the best among all algorithms. The improvement of our method compared to other algorithms is slight yet obtained by strict crossvalidation experiment.

\section{CONCLUSIONS}

Our team has raised a novel MS slice identification method. We processed the unbalanced dataset using SMOTE. Then, we used MBD to extract features, and proposed TSR-BBO method to train the classifier.

In the future, we shall try to collect more MS patients than used in this study. Advanced classifiers, such as convolutional neural network, stacked sparse autoencoder, may be used. Besides, we may use multi-atlas method ${ }^{68 / 69]}$ to perform a brain segmentation method.

\section{ACKNOWLEDGMENTS}

This study was supported by Natural Science Foundation of China (61602250), Open Research Fund of Hunan Provincial Key Laboratory of Network Investigational Technology (2016WLZC013), Open Fund of Key Laboratory of Symbolic Computation and Knowledge Engineering of Ministry of Education, Jilin University (93K172016K17), Natural Science Foundation of Jiangsu Province (BK20150983), Program of Natural Science Research of Jiangsu Higher Education Institutions (16KJB520025, 15KJB470010), Open fund for Jiangsu Key Laboratory of Advanced Manufacturing Technology (HGAMTL1601), Open fund of Key Laboratory of Guangxi High Schools Complex System and Computational Intelligence (2016CSCI01), Opening Project of State Key Laboratory of Digital Publishing Technology.

\section{REFERENCES}

1. A. Moroso et al., Cerebellar assessment in early multiple sclerosis, Cerebellum 16(2) (2017) 607-6112.

2. Y. Karaca et al., Rank determination of mental functions by 1D wavelets and partial correlation, J. Med. Syst. 41(1) (2017) 2.

3. Y. Karaca, The differential diagnosis of multiple sclerosis using convex combination of infinite Kernels, CNS Neurol. Disord. Drug Targets 16(1) (2017) 36-43.

4. J. A. N. Bijvank et al., A rare cause for visual symptoms in multiple sclerosis: Posterior internuclear ophthalmoplegia of Lutz, a historical misnomer, $J$. Neurol. 264(3) (2017) 600-602.

5. N. Ghasemi, S. Razavi and E. Nikzad, Multiple sclerosis: Pathogenesis, symptoms, diagnoses and cellbased therapy, Cell J. 19(1) (2017) 1-10.

6. L. S. Lens-Pechakova, Centenarian rates and life expectancy related to the death rates of multiple 
sclerosis, asthma, and rheumatoid arthritis and the incidence of type 1 diabetes in children, Rejuvenation Res. 19(1) (2016) 53-58.

7. M. Ingrisch et al., Dynamic contrast-enhanced magnetic resonance imaging suggests normal perfusion in normal-appearing white matter in multiple sclerosis, Invest. Radiol. 52(3) (2017) 135-141.

8. M. Pardini et al., The association between retinal nerve fibre layer thickness and $N$-acetyl aspartate levels in multiple sclerosis brain normal-appearing white matter: A longitudinal study using magnetic resonance spectroscopy and optical coherence tomography, Eur. J. Neurol. 23(12) (2016) 1769 1774 .

9. L. Wu, Classification of fruits using computer vision and a multiclass support vector machine, Sensors, 12(9) (2012) 12489-12505.

10. J. M. Sabater-Navarro et al., Computer vision haemorrhage detection, Br. J. Surg. 104 (2017) 24-24.

11. S. Wang and L. Wu, A novel method for magnetic resonance brain image classification based on adaptive chaotic PSO, Prog. Electromagn. Res. 109 (2010) 325-343.

12. V. Murray, P. Rodriguez and M. S. Pattichis, Multiscale AM-FM demodulation and image reconstruction methods with improved accuracy, IEEE Trans. Image Process. 19(5) (2010) 11381152 .

13. D. R. Nayak, R. Dash and B. Majhi, Brain MR image classification using two-dimensional discrete wavelet transform and AdaBoost with random forests, Neurocomputing 177 (2016) 188-197.

14. T. M. Zhan and Y. Chen, Multiple sclerosis detection based on biorthogonal wavelet transform, RBF Kernel principal component analysis, and logistic regression, IEEE Access 4 (2016) 7567-7576.

15. J. Yang, Pathological brain detection in MRI scanning via $\mathrm{Hu}$ moment invariants and machine learning, J. Exp. Theor. Artif. Intell. 29(2) (2017) $299-312$.

16. X.-X. Zhou, Comparison of machine learning methods for stationary wavelet entropy-based multiple sclerosis detection: Decision tree, $k$-nearest neighbors, and support vector machine, Simulation 92(9) (2016) 861-871.

17. M. F. Siddiqui, A. W. Reza and J. Kanesan, An automated and intelligent medical decision support system for brain MRI scans classification, PLoS One 10(8) (2015) e0135875.

18. P. Phillips, Z. Dong and J. Yang, Pathological brain detection in magnetic resonance imaging scanning by wavelet entropy and hybridization of biogeography-based optimization and particle swarm optimization, Prog. Electromagn. Res. 152 (2015) 41-58.
19. H. J. Fu et al., Characteristics of pore structure and fractal dimension of low-rank coal: A case study of lower Jurassic Xishanyao coal in the southern Junggar basin, NW China, Fuel 193 (2017) 254-264.

20. J. S. Lee, K. J. Wei and K. R. Wen, Image structure rebuilding technique using fractal dimension on the best match patch searching, Multimed. Tools Appl. 76(2) (2017) 1875-1899.

21. S. K. Kumar, R. Ganguli and D. Harursampath, Detecting width-wise partial delamination in the composite beam using generalized fractal dimension, Smart Struct. Syst. 19(1) (2017) 91-103.

22. Z. Arabasadi et al., Computer-aided decision making for heart disease detection using hybrid neural network-genetic algorithm, Comput. Methods Programs Biomed. 141 (2017) 19-26.

23. G. Ji, An MR brain images classifier system via particle swarm optimization and kernel support vector machine, Sci. World J. 2013 (2013) 130134.

24. S. Bahrami and F. D. Ardejani, Prediction of pyrite oxidation in a coal washing waste pile using a hybrid method, coupling artificial neural networks and simulated annealing (ANN/SA), J. Clean. Prod. 137 (2016) 1129-1137.

25. G. Yang, Automated classification of brain images using wavelet-energy and biogeography-based optimization, Multimed. Tools Appl. 75(23) (2016) 15601-15617.

26. Y. H. Lee, K. W. Kim and S. S. Kim, CUDA implementation of histogram stretching function for improving X-ray image, in 14th World Congress on Medical and Health Informatics. Ios Press, Copenhagen, Denmark; 2013, pp. 987-987.

27. X. Wu, Smart detection on abnormal breasts in digital mammography based on contrast-limited adaptive histogram equalization and chaotic adaptive real-coded biogeography-based optimization, Simulation 92(9) (2016) 873-885.

28. G. Wei, Color image enhancement based on HVS and PCNN, Science China Inform. Sci. 53(10) (2010) 1963-1976.

29. A. Gandhamal et al., Local gray level S-curve transformation - A generalized contrast enhancement technique for medical images, Comput. Biol. Med. 83 (2017) 120-133.

30. K. G. Lore, A. Akintayo and S. Sarkar, LLNet: A deep autoencoder approach to natural low-light image enhancement, Pattern Recognit. 61 (2017) 650-662.

31. M. Zieba, J. M. Tomczak and A. Gonczarek, RBMSMOTE: Restricted Boltzmann machines for synthetic minority oversampling technique, in 7th Asian Conference on Intelligent Information and Database Systems $(A C I I D S)$. (Springer, 2015, Bali, Indonesia), pp. 377-386. 
32. K. B. Pawar and S. L. Nalbalwar, Distributed Canny edge detection algorithm using morphological filter, in International Conference on Recent Trends In Electronics, Information \& Communication Technology (IEEE, Bengaluru, India, 2016), pp. 15231527.

33. Y. Jang, J. Mun and J. Kim, Resource-efficient FPGA architecture of canny edge detector, in International Society Design Conference (IEEE, Jeju, South Korea, 2016), pp. 299-300.

34. S. Liu et al., A fractal image encoding method based on statistical loss used in agricultural image compression, Multimed. Tools Appl. 75(23) (2016) $15525-15536$.

35. S. Liu, Distribution of primary additional errors in fractal encoding method, Multimed. Tools Appl. 76(4) (2017) 5787-5802.

36. J. J. Martin-Sotoca et al., New segmentation method based on fractal properties using singularity maps, Geoderma 287 (2017) 40-53.

37. J. B. Florindo, O. M. Bruno and G. Landini, Morphological classification of odontogenic keratocysts using Bouligand-Minkowski fractal descriptors, Comput. Biol. Med. 81 (2017) 1-10.

38. M. Liu et al., Distributional escape time algorithm based on generalized fractal sets in cloud environment, Chinese J. Electron. 24(1) (2015) 124-127.

39. C. Cattani and A. Ciancio, On the fractal distribution of primes and prime-indexed primes by the binary image analysis, Physica A 460 (2016) $222-229$.

40. C. Cattani and G. Pierro, On the fractal geometry of DNA by the binary image analysis, Bull. Math. Biol. 75(9) (2013) 1544-1570.

41. E. W. Dekking and F. M. Dekking, Helge von Koch's snowflake curve revisited, Am. Math. Mon. 123(2) (2016) 181-184.

42. X.-Q. Chen, Fractal dimension estimation for developing pathological brain detection system based on Minkowski-Bouligand method, IEEE Access 4 (2016) 5937-5947.

43. X. J. Yang et al., On a fractal LC-electric circuit modeled by local fractional calculus, Commun. Nonlinear Sci. Numer. Simul. 47 (2017) 200-206.

44. X. J. Yang, F. Gao and H. M. Srivastava, Exact travelling wave solutions for the local fractional twodimensional Burgers-type equations, Comput. Math. Appl. 73(2) (2017) 203-210.

45. J. A. T. Machado, D. Baleanu and C. Cattani, On exact traveling-wave solutions for local fractional Korteweg-de Vries equation, Chaos 26(8) (2016) 084312.

46. S. Lu and X. Qiu, A pathological brain detection system based on extreme learning machine optimized by bat algorithm, CNS Neurol. Disord. Drug Targets 16(1) (2017) 23-29.
47. T. Yuksel, Intelligent visual servoing with extreme learning machine and fuzzy logic, Expert Syst. Appl. 72 (2017) 344-356.

48. S. Du, Alzheimer's disease detection by pseudo zernike moment and linear regression classification, CNS Neurol. Disord. Drug Targets 16(1) (2017) 11-15.

49. Y. Chen, A feature-free 30-disease pathological brain detection system by linear regression classifier, CNS Neurol. Disord. Drug Targets 16(1) (2017) $5-10$.

50. A. Seal et al., UGC-JU face database and its benchmarking using linear regression classifier, Multimed. Tools Appl. 74(9) (2015) 2913-2937.

51. J. Yang, Identification of green, Oolong and black teas in China via wavelet packet entropy and fuzzy support vector machine, Entropy 17(10) (2015) 6663-6682.

52. J. Yang, Preclinical diagnosis of magnetic resonance (MR) brain images via discrete wavelet packet transform with Tsallis entropy and generalized eigenvalue proximal support vector machine (GEPSVM), Entropy 17(4) (2015) 1795-1813.

53. A. Liu, Magnetic resonance brain image classification via stationary wavelet transform and generalized eigenvalue proximal support vector machine, J. Med. Imaging Health Inform. 5(7) (2015) 13951403.

54. Z. Dong, Classification of Alzheimer disease based on structural magnetic resonance imaging by kernel support vector machine decision tree, Prog. Electromagn. Res. 144 (2014) 171-184.

55. J. Arias, J. A. Gamez and J. M. Puerta, Learning distributed discrete Bayesian network classifiers under mapreduce with Apache spark, Knowl.-Based Syst. 117 (2017) 16-26.

56. R. V. Rao, A. Liu and L. Wei, Abnormal breast detection in mammogram images by feed-forward neural network trained by jaya algorithm, Fundam. Inform. 151(1-4) (2017) 191-211.

57. J. Li, Texture analysis method based on fractional Fourier entropy and fitness-scaling adaptive genetic algorithm for detecting left-sided and right-sided sensorineural hearing loss, Fundam. Inform. 151(14) (2017) 505-521.

58. I. Aydogdu, Cost optimization of reinforced concrete cantilever retaining walls under seismic loading using a biogeography-based optimization algorithm with Levy flights, Eng. Optim. 49(3) (2017) 381400.

59. L. Wei, Fruit classification by wavelet-entropy and feedforward neural network trained by fitness-scaled chaotic ABC and biogeography-based optimization, Entropy 17(8) (2015) 5711-5728.

60. M. Khishe, M. R. Mosavi and M. Kaveh, Improved migration models of biogeography-based 
optimization for sonar dataset classification by using neural network, Appl. Acoust. 118 (2017) 15-29.

61. W. L. Lim et al., A biogeography-based optimization algorithm hybridized with tabu search for the quadratic assignment problem, Comput. Intell. Neurosci. (2016) 5803893.

62. Z. Hasan, K. Salman and M. E. El-Hawary, Linear quadratic regulator design for power system stabilizer using biogeography based optimization method, in IEEE Canadian Conference on Electrical And Computer Engineering (IEEE, New York, Vancouver, Canada, 2016).

63. B. Y. Bagde et al., Optimal network reconfiguration of a distribution system using biogeography based optimization, in 6th International Conference on Power Systems (ICPS) (IEEE, New Delhi, India, 2016), p. 6 .

64. Y.-K. Huo and G. Wei, An adaptive threshold for the Canny operator of edge detection, in International Conference on Image Analysis and Signal Processing (IEEE, Xiamen, China, 2010), pp. 371374.
65. I. Rossales and M. Luppe, Architecture for fractal dimension estimation based on minkowskibouligand method using integer distances, in 27th International Conference on Application-Specific Systems, Architectures and Processors (IEEE, London, England, 2016), pp. 231-232.

66. S. Liu et al., Numeric characteristics of generalized M-set with its asymptote, Appl. Math. Comput. 243 (2014) 767-774.

67. S. Liu et al., Fractal property of generalized M-set with rational number exponent, Appl. Math. Comput. 220 (2013) 668-675.

68. A. J. Asman et al., Multi-atlas learner fusion: An efficient segmentation approach for large-scale data, Med. Image Anal. 26(1) (2015) 82-91.

69. Y. Huo et al., Consistent cortical reconstruction and multi-atlas brain segmentation, Neuroimage 138 (2016) 197-210. 